\title{
On the Use of the Word "Absolute" in Pollen Statistics
}

Ever since Margaret Davis (1963) taught us to calculate rates of pollen influx, we pollen people have been tempted to remove ourselves from the dubious statistical heritage of our percentage diagrams. Once all pollen diagrams were percentage diagrams. We had to express the population history of a pollen taxon in terms of the summed fluctuations of all other taxa. We were uneasy about doing this, and our mathematical friends assured us that we were right to be uneasy (Mosimann, 1963). But Davis told us not to despair. If only we worked hard enough to count all the pollen grains in unit volume of sediment, we could divide the result by the sediment accumulation rate and derive independent statistics for each pollen taxon. We were being taught to draw pollen influx diagrams, but this term was slow in coming. At first we were inclined to emphasize the merits of the new diagrams and to distinguish them from those shameful percentage diagrams of the past by calling the new thing the absolute pollen diagram.

But we can only calculate the pollen influx for this new diagram when the sediment accumulation rate is known, and it is remarkable how seldom we can be certain that the sedimentation rate has not changed over some critical interval. Though we offer prayers to radiocarbon, varve-like bands, remanent magnetism, the isotopes of lead, or the date of the Ambrosia rise, we find our prayers answered with an uncertain voice. Then we cannot calculate reliable sediment accumulation rates and, hence, cannot construct a pollen influx diagram. But all that hard work of estimating grains per unit volume may have been done, and it is a pity to lose the glory of it. So we incline to pub- lishing diagrams of pollen per unit volume or mass. And we put a bold face on our necessity by calling these diagrams "absolute" too.

But there is nothing "absolute" about a statistic that expresses numbers of pollen grains as a function of water, minerals, or detritus, all of which have been contributed in unknown proportions that may well change from interval to interval. Pollen diagrams of this kind are relative diagrams. They represent pollen relative to concentrations of clastic or detrital materials. To call them "absolute" is absurd.

There may be paleoecological investigations in which it is useful to examine how a pollen taxon fluctuates relative to sediment volume or mass. This might be so, for instance, if there were good reasons for believing that the sediment rate was nearly constant over an interval even though it could not be measured, and there may be times when pollen might be used in studies of sedimentation itself. For these studies it would be well to have a descriptive term which would immediately reveal the kinds of pollen data being used. R. B. Davis has suggested to me the pollen concentration diagram. This term would be defined as describing all pollen diagrams in which numbers of pollen grains are measured and scaled in units of mass or volume of sediment. It should be a requirement of journal editors that these diagrams always include clear statements of the units of mass or volume used.

The pollen concentration diagram is likely to be useful in studies of dispersal of pollen in the ocean basins and in large lakes, where reasonable models of sediment 
transport are available, and the goal of the research is the detection of long-distance transport of pollen. For these studies, the new term should serve well, for the results are likely to be read by oceanographers and others not sensitive to the pollen analysist's parochial definitions of "absolute."

Pollen concentration diagrams ought not to survive long in studies of bogs because growth and accumulation of peat is inherently liable to large changes over short intervals. Indeed, the difficulty of arriving at good peat accumulation rates may be so great that pollen influx diagrams are likely to continue to be rare for bogs.

We now have three different ways of expressing pollen data: the pollen percentage diagram, the pollen influx diagram and the pollen concentration diagram. Only the in- flux diagram has anything absolute about it, and its absoluteness is heavily qualified by the accuracy of the estimate of sedimentation rate. It is time that journal editors forbade pollen analysts to call their diagrams "absolute." Absolutely.

\section{REFERENCES}

Davis, M. B. (1963) On the theory of pollen analysis. American Journal of Science, 261, 897-912.

Mosimann, J. E. (1963) On the compound negative multinomial distribution and correlations among inversely sampled pollen counts. Biometricka $\mathbf{5 0}$, $47-54$.

Paul A. Colinvaux

Department of Zoology

The Ohio State University

1735 Neil Avenue

Columbus, Ohio 43210 